\title{
Efficacy of vaccination on Staphylococcus aureus and coagulase-negative staphylococci intramammary infection dynamics in 2 dairy herds
}

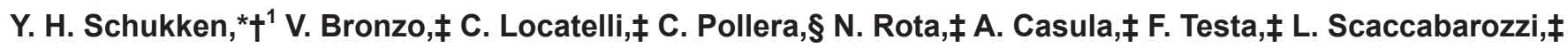 \\ R. March,\# D. Zalduendo,\# R. Guix,\# and P. Moroni* $¥$ \\ *Department of Population Medicine and Diagnostic Sciences, College of Veterinary Medicine, Cornell University, Ithaca, NY 14853 \\ †GD Animal Health, Arnsbergstraat 7, 7418 EZ Deventer, the Netherlands \\ fUniversità degli Studi di Milano, Dipartimento di Scienze Veterinarie per la Salute, la Produzione Animale e la Sicurezza Alimentare, \\ via Celoria 10, 20133 Milan, Italy \\ §Università degli Studi di Milano, Dipartimento di Scienze Veterinarie e Sanità Pubblica, via Celoria 10, 20133 Milan, Italy \\ \#Hipra S. A. Laboratorios, Avenida La Selva 135, Amer (Girona), Spain
}

\begin{abstract}
The aim of this study was to evaluate vaccine efficacy of a commercial vaccine (Startvac, Hipra Spain) aimed at reducing intramammary infections (IMI) with Staphylococcus aureus and coagulase-negative staphylococci under field conditions. During the 21-mo duration of the study, 1,156 lactations from 809 cows were enrolled in 2 herds. During the first phase of the trial, all cows that were due to calve were vaccinated until approximately $50 \%$ of cows in the milking herd were vaccinated (at $\sim 6$ mo). At that point, when $50 \%$ vaccination coverage was reached, cows that were due to calve were randomly assigned to be vaccinated or left as negative controls. Cure rate, rate of new infection, prevalence, and duration of infections were analyzed. Vaccination resulted in a moderate reduction in incidence of new staphylococcal IMI and a more pronounced reduction in duration of IMI associated with reduction of the basic reproduction ratio of Staph. aureus by approximately $45 \%$ and of coagulase-negative staphylococci by approximately $35 \%$. The utilization of vaccine in combination with other infection-control procedures, such as excellent milking procedures, treatment, segregation, and culling of known infected cattle, will result in an important reduction in incidence and duration of intramammary staphylococcal infections.
\end{abstract}

Key words: Staphylococcus aureus, coagulase-negative staphylococci, intramammary infection, vaccine

\section{INTRODUCTION}

Mastitis is one of the most frequently occurring and costly diseases in dairy cows (Barkema et al., 2006;

Received February 2, 2014.

Accepted April 15, 2014.

${ }^{1}$ Corresponding author: y.schukken@gddeventer.com
Halasa et al., 2007). Clinical mastitis is characterized by visible changes in milk, including the presence of clots, flakes, serum, or even blood inclusion. Subclinical mastitis is characterized by increased SCC, reduced milk production, and, in many cases, a higher risk of early removal from the farm. Several preventative strategies have been applied to minimize the incidence of bovine mastitis, including optimization of milking procedures and milking hygiene, antibiotic therapies, vaccinations, segregation, and culling of persistently infected cows. However, mastitis remains an important disease on many dairy farms and, due to the high costs of clinical mastitis, reduction in the severity of the symptoms of mastitis and obtaining a more rapid clearance of established infections is of great value to dairy farmers (Cha et al., 2011; Hertl et al., 2011). The severity of clinical symptoms of coliform mastitis has been shown to be reduced by immunization with commercially available J-5 bacterin (Wilson et al., 2007). The efficacy of this vaccine for the prevention of mastitis caused by Escherichia coli has been investigated in experimental challenge studies (Wilson et al., 2007). These studies implied that immunization with J-5 bacterin reduced the severity of local and systemic signs of clinical mastitis following intramammary challenge. Efficacy of vaccination against Staphylococcus aureus and CNS is a very different concept than efficacy of vaccination against $E$. coli (Torvaldsen and McIntyre, 2002). Whereas with $E$. coli the vaccine is mostly expected to reduce severity of infection, with Staph. aureus and CNS the vaccine is particularly valuable when vaccination results in a reduction of incidence and duration of infection, the key contributors to within herd infection dynamics (Schukken et al., 2011).

Vaccines against staphylococci have been studied and suggested as an important tool in the management of staphylococcal infections in dairy cows (Pereira et al., 2011; Daum and Spellberg, 2012). Experimental chal- 
lenge studies with Staph. aureus have shown an effect of vaccination on the amount of bacterial shedding after challenge (Pérez et al., 2009); however, such experimental studies were not able to demonstrate a reduction in infection transmission. Several study designs to estimate vaccine efficacy of contagious infections have been proposed (Haber et al., 1991; Halloran et al., 1991, 1997, 1998). Randomization can take place at either the herd or at the individual animal level. To estimate the overall population vaccine efficacy using herd-level randomization, large numbers of vaccinated and control herds would be necessary. Within-herd randomization of cows to vaccination and control has obvious study size benefits, but is hampered by the potential herd immunity provided by vaccinates to the control animals in the same herd (Halloran et al., 1991). Comingling of vaccinated and control cows allows the calculation of direct vaccine efficacy, but this estimate of vaccine efficacy will be biased toward zero. This direct vaccine efficacy is an underestimation of the overall population vaccine efficacy due to the herd immunity of the vaccinated individuals that protect the unvaccinated controls (Halloran et al., 1991). However, instead of basing vaccine efficacy on infection incidence, vaccine efficacy can be estimated based on infection transmission and infection duration parameters (Halloran et al., 1997). These infection dynamics parameters can be estimated from precisely documented infections in comingled populations, and the resulting vaccine efficacy turns out to be an unbiased estimate of overall population vaccine efficacy as long as the analysis is controlled for total exposure experience (Lu et al., 2009).

The number of vaccines against staphylococcal pathogens available on the market is small, and the efficacy of the results of these in peer-reviewed studies from commercial dairy farms is generally limited (Middleton et al., 2009). Recently, a combined staphylococcal and J5 E. coli vaccine (Startvac, Hipra Spain), was introduced in the European market and, subsequently, in many other countries worldwide. The staphylococcal component of the vaccine is based on a bacterin of Staph. aureus strains with particular high cell wall components, such as exopolysaccharides, that may be involved in the biofilm phenotype of the bacteria (Harro et al., 2010; Prenafeta et al., 2010).

To evaluate vaccine efficacy in the case of Staph. aureus and CNS infections, the infection status of quarters of cows needs to be determined precisely over time (Halloran et al., 1997). Such precise data will allow the evaluation of vaccination on new IMI and IMI duration; at this point, few, if any, such studies have been reported in the literature. The objective of the current trial was to evaluate vaccine efficacy under field condi- tions in 2 herds with a known infection prevalence of Staph. aureus and CNS.

\section{MATERIALS AND METHODS}

\section{Herds}

To evaluate vaccine efficacy we studied infection dynamics in 2 herds with a total of approximately 450 dairy cows milking at any point in time. The herds had a known prevalence of Staph. aureus of at least $5 \%$ of cows and a bulk milk SCC between 250,000 and 400,000 cells/mL. Both herds used dry cow therapy on all quarters of all cows. Clinical mastitis cases were treated according to herd-specific protocols that were similar but not identical. Herd A had 2 dedicated milkers that used a milking protocol with forestripping and wiping with single-use cloth towels. Herd B had one dedicated milker that used forestripping and wiping with single-use paper towels. Both herds used postmilking teat disinfection. Culling decisions were made by the farm owners based on fertility and lameness criteria in both herds.

The trial started in May 2011, with sampling, vaccinating, and collection data gathered on the farms until February 2013 for farm A, for a total of $21 \mathrm{mo}$, and October 2012 for farm B, for a total of 18 mo. Farm A maintained an average of 130 Holstein milking cows housed in freestall barns in deep-bedded cubicles with straw. Farm B maintained an average of 320 Holstein milking cows housed in freestall barns in deep-bedded cubicles with sawdust. On both farms, cows that were close to calving were moved to a loose-housing maternity pen bedded with straw. Animals were housed for the first week of lactation in a large loose-housing pen with straw. After 1 wk of lactation, cows were moved to freestall facilities. All groups of cows in both dairies were fed a balanced TMR in feed alleys with headlocks that allowed restraint of cows for examination and administration of treatments, medications, and vaccinations. No segregation of cows based on IMI status or SCC level was done on either farm.

\section{Milking Equipment Evaluation}

On Farm A, cows were milked in a double-12 parallel parlor 2 times per day, whereas Farm B had a double-15 herringbone parlor and cows were also milked 2 times per day. On the farms, milking equipment was evaluated twice during the study period by technicians of the Regional Breeding Association using a complete ISO 6690:2007-defined evaluation (ISO, 2007). Equipment evaluation took place at the beginning and at approxi- 
mately 1 yr into the study. No important concerns with milking equipment were identified on either farm.

\section{Cow Data}

Cow data on calving, parity, reproduction (AI dates, pregnancy), clinical disease (including retained placenta, endometritis, metritis, lameness, clinical mastitis, and metabolic diseases such as ketosis, abortion, and displaced abomasum), and culling was collected for all cows in the herd. During the trial, Italian DHIA testing in both herds was done monthly for milk production, fat, protein, and SCC, but these data were not further analyzed for this report. All breedings on both farms were done using AI. Cow data were collected using a computerized herd record-keeping system (Dairy Comp 305, Valley Agricultural Software, Tulare, CA).

\section{Vaccination}

Vaccination took place according to label directions in the dry period and early lactation. The first vaccination was at $45 \mathrm{~d}( \pm 3 \mathrm{~d})$ before the expected parturition date, the second vaccination at $35 \mathrm{~d}$ thereafter $( \pm 3 \mathrm{~d})$, corresponding to $10 \mathrm{~d}$ before the expected parturition date, and the third vaccination was at $52 \operatorname{DIM}( \pm 3 \mathrm{~d})$. No placebo or sham vaccination was used in this trial. Cows going through a second dry period during the study were kept in the same treatment group (vaccinated or control). At the start of the trial, all cows that were due to calve were vaccinated until approximately $50 \%$ of cows in the milking herd were vaccinated $(\sim 6$ mo). At that point in time, when $50 \%$ vaccination coverage was reached, cows were randomly assigned to be vaccinated or left as controls. Trained farm personnel on farm $\mathrm{A}$ and the herd veterinarian on farm B performed all vaccinations. Assignment of vaccination was done using the European cow registration number, whereby even-numbered cows were vaccinated and oddnumbered cows were kept as controls. Cows were identified in each farm using unique farm-specific ear tags. No logical relationship existed between the on-farm ear tag number and the official 13-digit European cow registration number. We thereby assume that this was essentially a randomized controlled and single-blinded trial, as the herd staff was not aware of the vaccination status of the animals.

\section{Milk Sampling}

Monthly quarter sampling of all lactating cows in herds was done during the trial period. In addition, quarters were sampled by the farm staff when a case of clinical mastitis occurred, when cows were dried off, upon calving, and at culling. Samplings related to dry off, calving, and culling were done within $24 \mathrm{~h}$ of the event. Sampling in cases of clinical mastitis was done upon detection, before treatment was applied. Before sampling, teat ends were carefully cleaned and disinfected with chlorhexidine. First streams of foremilk were discharged, and then approximately $10 \mathrm{~mL}$ of milk was collected aseptically from each teat into sterile vials. Samples were stored at $4^{\circ} \mathrm{C}$ until bacteriological assays and SCC tests were initiated immediately after arrival back in the laboratory.

\section{Bacteriological Analysis}

Bacteriological cultures were performed according to standards of the National Mastitis Council (NMC, 1999). Ten microliters of each milk sample were spread on blood agar plates ( $5 \%$ defibrinated sheep blood). Plates were incubated aerobically at $37^{\circ} \mathrm{C}$ and examined after $24 \mathrm{~h}$.

Colonies were provisionally identified on the basis of morphology, hemolysis patterns, and Gram staining. Gram-positive organisms were differentiated in staphylococci and streptococci by the catalase reaction. The coagulase tube test in rabbit plasma was used to differentiate Staph. aureus and CNS species. Catalase- and coagulase-positive bacteria were reported as Staph. aureus, whereas catalase-positive and coagulase-negative species were reported as CNS. Catalase-negative organisms had their identity confirmed by the API20Strep system (bioMerieux, Marcy l'Etoile, France), designed for Streptococcus spp. identification. Pathogens reported as other Streptococcus spp. corresponded to species of streptococci that are less commonly reported in the literature or to pathogens that are not included in the API system identification panel. Gram-negative bacteria were identified by oxidase test, as well as by growth characteristics onto MacConkey agar (Oxoid Ltd., Basingstoke, UK) and Eosin Methylene Blue agar (Oxoid Ltd.; http://www.oxoid.com/UK/blue/prod_ detail/prod_detail.asp?pr=CM0069\&org=66). Further identification was performed with the API20E and API20NE system (bioMerieux, Marcy l'Etoile, France). Gram-negative bacteria with very low prevalence that could not be identified by the methods described were reported as "other gram-negative." The numbers of each colony type were recorded. Representative colonies were then subcultured on blood agar plates and incubated again at $37^{\circ} \mathrm{C}$ for $24 \mathrm{~h}$ to obtain pure cultures. For plates with Staph. aureus and CNS growth, the number of colonies was recorded for each species isolated, and colonies were reisolated and frozen for future characterization at $-80^{\circ} \mathrm{C}$ in Nutrient Broth (Merck KGaA, Darmstadt, Germany) with $15 \%$ glycerol. 


\section{Definition of Infection Status}

Staphylococcus aureus was considered to cause an IMI if at least 1 colony $(\geq 100 \mathrm{cfu} / \mathrm{mL})$ was isolated. For CNS, IMI was defined by the isolation of at least 2 colonies $(\geq 200 \mathrm{cfu} / \mathrm{mL})$ from a single sample or $\geq 100$ $\mathrm{cfu} / \mathrm{mL}$ from a clinical sample. When multiple (at least 2 out of 3 ) consecutive samples with $\geq 100 \mathrm{cfu} / \mathrm{mL}$ of CNS were identified, this was also considered an IMI. These definitions are based on the consensus opinion of mastitis research workers as published by Dohoo et al. (2011) and Andersen et al. (2010). A quarter was defined as uninfected and at risk for a new infection when 2 consecutive samples were culture-negative. An infection was considered cured if 2 consecutive monthly milk samples did not show the presence of the causative organism. Milk samples where 3 or more species were identified were considered contaminated. All culture results were kept from both farm staff and herd veterinarians until the very end of the study. When entering or leaving the trial, or reentering after calving, a single negative sample was considered sufficient to be defined as uninfected.

\section{Statistical Analysis}

Data were analyzed using the SAS version 9.2 system (SAS Institute Inc., Cary, NC). Descriptive analysis was done on all important outcome variables and covariates. Transformations were used where outcome variables were not normally distributed (e.g., SCC and cfu).

Logistic Regression Analysis-Risk Factors for New IMI and Cure of IMI. Linear regression models were used for analysis of crude prevalence and incidence of IMI. In these generalized linear models the only data were used after the 50/50 randomization in the herds had started. Every quarter-month at risk of either an incident or prevalent staphylococcal IMI contributed a line of data to this analysis. The generalized linear model had the following format:

$$
\begin{gathered}
\text { Logit }(\mathrm{Y}) \text { = intercept + MIM + lactgroup } \\
+ \text { herd + vaccination + complex error }
\end{gathered}
$$

where $\mathrm{Y}$ is the outcome of interest (incidence or prevalence of Staph. aureus and CNS); MIM is months in milk; lactgroup is the lactation number of the cow, grouped into 1, 2, and 3+; herd is the herd code; and vaccination is either vaccinated or control. Complex error is a correlated error term where within-cow correlation is combined with a random binomial error. Relevant interactions were evaluated in the model and included when statistically significant.
Duration of infection was estimated with the use of time-to-event analysis. Kaplan-Meier estimates of the survivor curves were used for graphical representation of the results. Cox regression was used for estimating the effect of vaccination on the duration of infection. For this analysis, only new infections were used that started after the 50/50 randomization in the herds had started.

Modeling Infection Dynamics. The rate of new infections per day at risk was calculated for vaccinated and control cows. The rates were calculated on a monthly basis (calendar months) for the duration of the trial. For evaluation of vaccine efficacy of Staph. aureus and CNS, the transmission rate $(\beta)$, taking exposure into account, was calculated and compared between vaccinated and control cows. Exposure was based on the number of Staph. aureus- or CNS-shedding quarters at the same time in the herd. No distinction was made between infected quarters in the same cow and the susceptible quarters and infected quarters in other cows. The modeled relationship was defined as

$$
\begin{gathered}
\text { New Staph. aureus or CNS infections }(\mathrm{v} / \mathrm{c}) \\
\beta_{(\mathrm{v} / \mathrm{c})} \times \mathrm{S}_{(\mathrm{v} / \mathrm{c})} \times\left(\mathrm{I}_{\mathrm{v}}+\mathrm{I}_{\mathrm{c}}\right)+\text { covariates }
\end{gathered}
$$

where $\mathrm{v} / \mathrm{c}$ is vaccinated or control; $\mathrm{S}$ is the number of susceptible quarters; I is the number of infected quarters; and $\beta$ is the transmission parameter. Vaccine efficacy for new infections may then be estimated as $1-\left(\beta_{\mathrm{v}} / \beta_{\mathrm{c}}\right)$.

Similarly, cure of infection was modeled using

$$
\begin{aligned}
& \text { Cure Staph. aureus or CNS infections } \mathrm{v}_{\mathrm{v} / \mathrm{c})}= \\
& \qquad \alpha_{(\mathrm{v} / \mathrm{c})} \times \mathrm{I}_{(\mathrm{v} / \mathrm{c})}+\text { covariates, }
\end{aligned}
$$

where $\alpha$ is the cure rate of infections. Again, vaccine efficacy may then be estimated as $1-\left(\alpha_{v} / \alpha_{c}\right)$. Estimates of $\alpha$ and $\beta$ were obtained through linear models using Poisson regression (see also Lam et al., 1996; Barlow et al., 2013). The regression model for estimation of $\beta$ was

$$
\ln \left(\text { no. of new infections } \mathrm{v} / \mathrm{c}_{\mathrm{c}}\right)=\mathrm{b}_{\mathrm{v} / \mathrm{c}}^{*}+\text { covariates }+ \text { offset, }
$$

where the offset is given by $\ln \left\{\left[\mathrm{S}_{\mathrm{v} / \mathrm{c}} \times\left(\mathrm{I}_{\mathrm{v}}+\mathrm{I}_{\mathrm{c}}\right)\right] / \mathrm{N}\right\}$, where $\mathrm{N}$ is the total population size. The parameter $\beta$ can then be calculated as $\exp \left(\beta^{*}\right)$. For estimation of $\alpha$, the Poisson regression equation was

$\ln \left(\right.$ no. of cured infections $\left.\mathrm{v}_{\mathrm{c}}\right)=\mathrm{a}_{\mathrm{v} / \mathrm{c}}^{*}+$ covariates + offset,

where the offset is given by $\operatorname{Ln}\left(I_{v / c}\right)$. The parameter $\alpha$ was then calculated as $\exp \left(\alpha^{*}\right)$. 
The unit of analysis in both the regression analysis to estimate $\beta_{\mathrm{v} / \mathrm{c}}^{*}$ and $\alpha_{\mathrm{v} / \mathrm{c}}^{*}$ was a calendar month. All data were used in this analysis, and a covariate that measured the month to or since the $50 / 50$ vaccination point was included as a covariate in the model.

Population vaccine efficacy was estimated using the parameters $\alpha$ and $\beta$, where vaccine efficacy were respectively defined as

$$
\text { Vaccine efficacy for new infections }=1-\frac{\beta_{\text {vaccinated }}}{\beta_{\text {control }}},
$$

whereas

$$
\text { Vaccine efficacy for cure of infections }=1-\frac{\alpha_{\text {control }}}{\alpha_{\text {vaccinated }}} \text {. }
$$

Combining the information of parameters $\alpha$ and $\beta$ into an overall infection reproduction ratio provides an unbiased summary parameter on vaccine efficacy. The basic reproduction ratio $\left(\mathbf{R}_{\mathbf{0}}\right)$ was defined as $\mathbf{R}_{0}=\beta / \alpha$, and the resulting vaccine efficacy is then calculated as

$$
1-\frac{R_{0, \text { vaccinated }}}{R_{0, \text { control }}}=1-\frac{(\beta / \alpha)_{\text {vaccinated }}}{(\beta / \alpha)_{\text {control }}} .
$$

The variance of $R_{0}$ may be calculated from the sum of the variance of the logarithm of the 2 components of $\mathrm{R}_{0}: \operatorname{Var}\left[\ln \left(\mathrm{R}_{0}\right)\right]=\operatorname{Var}\left(\beta^{*}\right)+\operatorname{Var}\left(\alpha^{*}\right)$. This overall efficacy parameter is expected to provide the best summary of the overall effect of vaccination on infection dynamics in a vaccinated population (Halloran et al., 2008).

\section{Samples Size}

The study was planned using a design of comingling vaccinates and controls with 1 control per vaccinate. As cow is the unit of vaccination, sample size calculations were performed at cow level. Prior data indicated that the new infection rate among controls is approximately 0.15 per lactation. This new infection risk of 0.15 includes both Staph. aureus and CNS infections. If the true vaccine efficacy is at least $50 \%$, then the new infection rate for vaccinated cows is 0.075 (Dohoo, 2004). We needed to study at least 250 vaccinated cows and 250 control cows to be able to reject the null hypothesis that the new infection rates for vaccinated and control cows were equal (efficacy $=0$ ) with probability (= power) 0.8 . The Type I error probability associated with this test of this null hypothesis is 0.05 . Because of the within-cow dependency due to comingling (Hal- loran et al., 1997), we estimated an increased sample size by approximately $25 \%$ resulting in at least 315 cows per treatment arm, resulting in a study size of at least 630 cows in total.

\section{RESULTS}

\section{Data Quality}

Data checks and data entry occurred throughout study. Entry into the vaccination group was not as fast as expected on farm A, as pregnant heifers were initially not vaccinated. This was corrected in the database as soon as it was noted. For this reason, the farm reached the 50/50 point a few months later; thus, it was decided to keep the herd in the study for a longer period compared with farm B. Data quality was checked throughout the study and additional information on incomplete data points was collected where needed. Vaccination compliance was not always perfect during the trial; this is discussed in more detail herein.

\section{Descriptive Statistics}

During the entire study, a total of 1,156 lactations in 809 cows were identified in both herds; 658 cows (56.92\%) were enrolled as controls, 343 cows $(29.67 \%)$ were fully vaccinated, and 155 cows $(13.34 \%)$ started the vaccination but were not fully vaccinated due to calving date estimation errors in pregnancy checking, early pregnancy loss, abortions, early calving, or end of the study. As vaccination was initially done on all cows calving into the lactating herd, the percentage of cows that were vaccinated increased rapidly in both herds. The percentage of vaccinated lactations in each herd throughout the trial is shown in Figure 1. In herd $\mathrm{B}$, the $50 / 50$ status was reached in mo 8 of the study, whereas in herd A this was at 11 mo into the study. Given that vaccinations start approximately 2 mo before anticipated calving, the change in randomization procedure started in herd B at 6 mo after the start of the study, whereas this was 9 mo after the start of the study in herd A.

\section{Bacterial Culture Results}

Throughout the study, 39,506 quarter milk samples were taken and used for bacterial culture. The results of bacterial culture of all these samples are shown in Table 1. The most commonly isolated pathogens in herd A were Staph. aureus $(2,151 ; 15.6 \%)$ and CNS (937; 6.8\%). In contrast, in herd B, CNS $(1,139 ; 4.6 \%)$ were more frequently identified then Staph. aureus $(929 ; 3.8 \%)$. Culture-negative status was observed in 


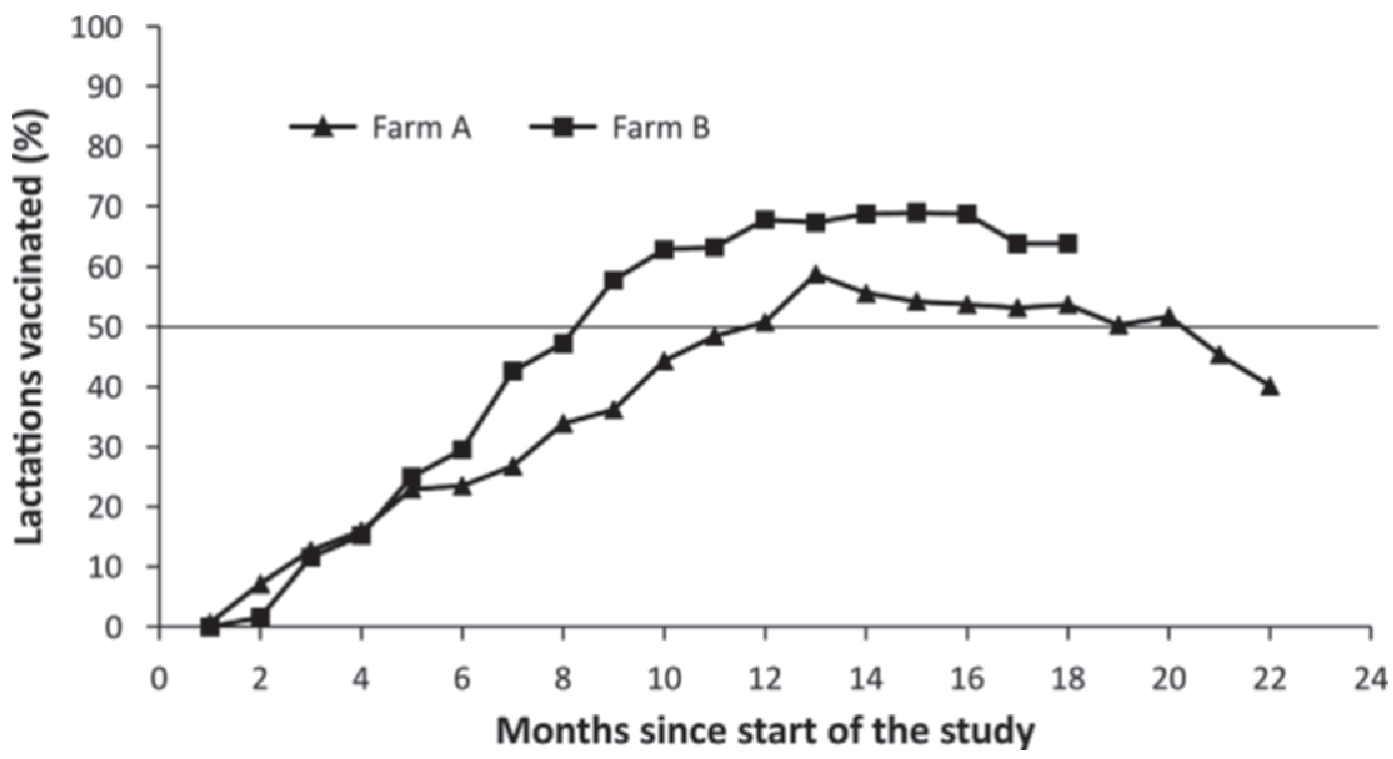

Figure 1. Percentage of lactations that were either vaccinated or control. In herd A the 50/50 status was reached in mo 11 into the study, whereas in herd $\mathrm{B}$ this was at 8 mo.

9,503 samples (69\%) for farm A and in 19,936 samples (80.5\%) for farm B. Prevalence of Staph. aureus during the course of the study remained more or less stable in farm A, ranging from $19.6 \%$ at mo 1 to $14.8 \%$ at mo 22 , but reduced dramatically in farm B from $10.5 \%$ at mo 1 to $1.2 \%$ at mo 18 .
In both farms, a fairly stable situation existed, without much change in prevalence of CNS IMI, ranging from $5.0 \%$ at mo 1 to $9.2 \%$ at mo 22 for farm A and from $5.1 \%$ at mo 1 to $4.4 \%$ at mo 18 for farm B. When expressing prevalence by month in lactation, the data indicated a gradually increasing difference in

Table 1. Bacterial results of all samples collected during the trial, monthly samples, dry off, calving, culling, and clinical mastitis cases

\begin{tabular}{|c|c|c|c|c|}
\hline \multirow[b]{2}{*}{ Pathogen } & \multicolumn{2}{|c|}{ Farm A } & \multicolumn{2}{|c|}{ Farm B } \\
\hline & No. & Percent & No. & Percent \\
\hline Staphylococcus aureus & 2,151 & 15.6 & 929 & 3.8 \\
\hline CNS & 937 & 6.8 & 1,139 & 4.6 \\
\hline Streptococcus bovis & 0 & 0.0 & 50 & 0.2 \\
\hline Streptococcus canis & 1 & 0.0 & 4 & 0.0 \\
\hline Streptococcus dysgalactiae & 19 & 0.1 & 176 & 0.7 \\
\hline Streptococcus mitis & 14 & 0.1 & 36 & 0.1 \\
\hline Streptococcus uberis & 132 & 1.0 & 217 & 0.9 \\
\hline Streptococcus spp. & 89 & 0.6 & 117 & 0.5 \\
\hline Corynebacterium spp. & 40 & 0.3 & 63 & 0.3 \\
\hline Enterococcus faecalis & 38 & 0.3 & 55 & 0.2 \\
\hline Lactococcus lactis & 11 & 0.1 & 70 & 0.3 \\
\hline Aerococcus viridans & 58 & 0.4 & 88 & 0.4 \\
\hline Escherichia coli & 81 & 0.6 & 191 & 0.8 \\
\hline Enterobacter spp. & 19 & 0.1 & 17 & 0.1 \\
\hline Other gram-negative pathogen & 52 & 0.4 & 36 & 0.1 \\
\hline Klebsiella spp. & 6 & 0.0 & 116 & 0.5 \\
\hline Pasteurella spp. & 2 & 0.0 & 8 & 0.0 \\
\hline Proteus spp. & 63 & 0.5 & 65 & 0.3 \\
\hline Prototheca spp. & 3 & 0.0 & 0 & 0.0 \\
\hline Serratia spp. & 4 & 0.0 & 15 & 0.1 \\
\hline Trueperella pyogenes & 0 & 0.0 & 2 & 0.0 \\
\hline Bacilli & 5 & 0.0 & 26 & 0.1 \\
\hline Blind quarter & 260 & 1.9 & 539 & 2.1 \\
\hline Missing or contaminated & 671 & 2.0 & 1,452 & 3.5 \\
\hline Culture negative & 9,503 & 69.0 & 19,936 & 80.5 \\
\hline Total & 14,159 & 100 & 25,347 & 100 \\
\hline
\end{tabular}



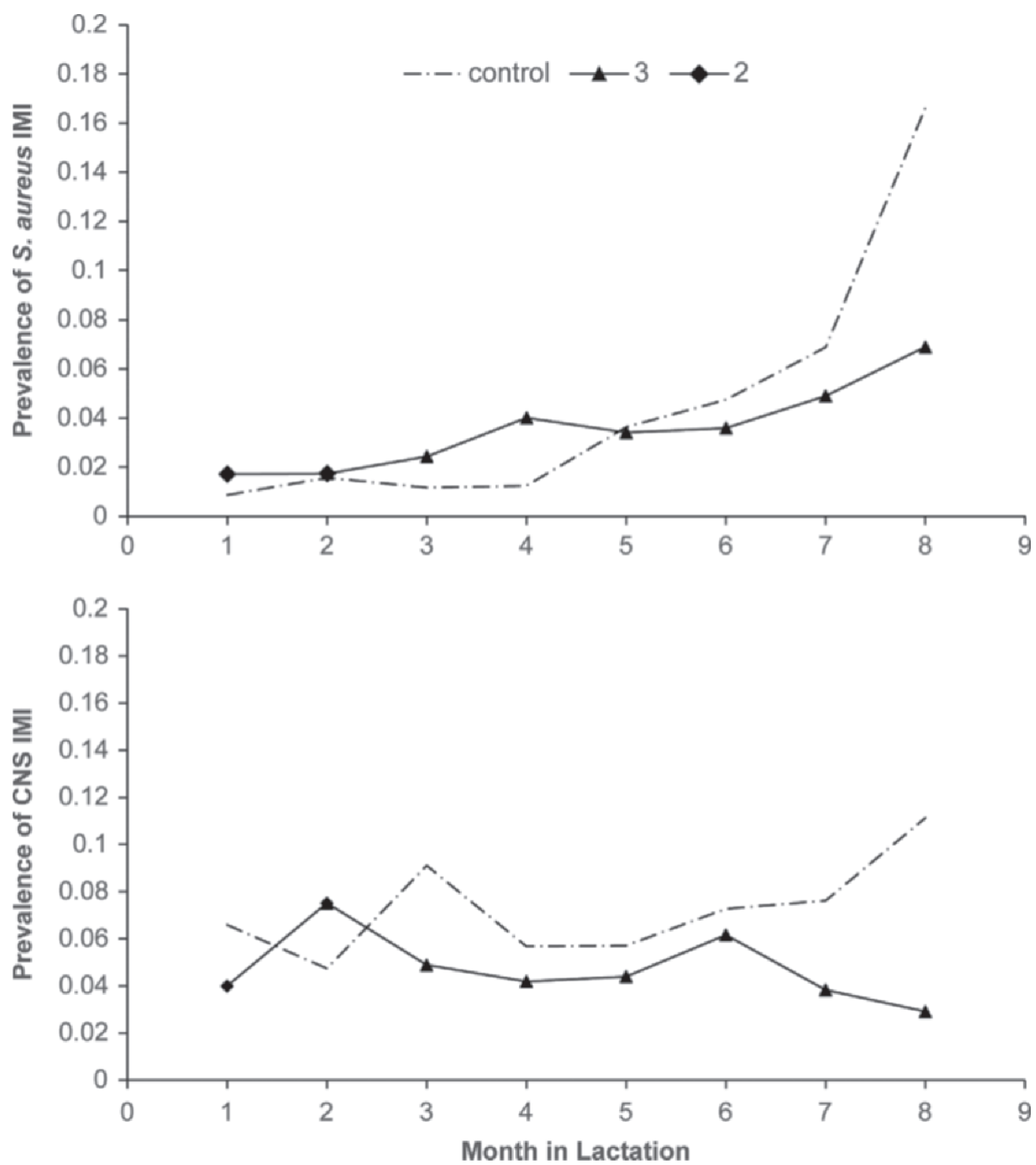

Figure 2. Prevalence of Staphylococcus aureus (top) and CNS (bottom) IMI in all quarters during the course of the study in vaccinated and control (dash-dotted line) cows. Only cows that were eventually fully vaccinated were included in this analysis. As per vaccination protocol, vaccinated cows received 2 vaccinations at the start of lactation (2) and received the third and final dose (3) at approximately 53 DIM.

prevalence between controls and vaccinates. This trend was present and statistically significant for both Staph. aureus and CNS IMI. The least squares means of the prevalence of infection for Staph. aureus and CNS is shown in Figures 2a and 2b.

\section{Statistical Analysis}

Logistic Regression and Cox Regression Analysis-Risk Factors for New IMI and Cure of IMI. Risk of new IMI with Staph. aureus and CNS was ana- lyzed by generalized linear regression analysis analyzing only new infections that occurred in cows calving after the 50/50 randomization had started. The final logistic regression models are shown in Table 2. For both Staph. aureus and CNS, new infections risk was not significantly affected by vaccination $(P>0.05)$ when evaluated as a main effect. For Staph. aureus, new infections increased with increasing DIM, increasing parity, and having a history of a previous Staph. aureus infection. Regression of new CNS infections showed a significant interaction between month in lactation and vaccination, 
Table 2. Final logistic regression models of new Staphylococcus aureus and CNS IMI ${ }^{1}$

\begin{tabular}{|c|c|c|c|c|c|}
\hline Effect & & \multicolumn{2}{|c|}{ Staph. aureus } & \multicolumn{2}{|c|}{ CNS } \\
\hline Intercept & & $-3.48(1.45)$ & 0.02 & $-3.56(0.52)$ & $<0.001$ \\
\hline \multirow[t]{5}{*}{ Month in lactation } & 1 & $-1.24(0.38)$ & 0.00 & $-0.13(0.38)$ & 0.73 \\
\hline & 2 & $-0.90(0.36)$ & 0.01 & $0.91(0.33)$ & 0.01 \\
\hline & 3 & $-0.68(0.35)$ & 0.05 & $0.28(0.36)$ & 0.44 \\
\hline & 7 & $0.19(0.40)$ & 0.64 & $-0.02(0.04)$ & 0.96 \\
\hline & 8 & $0.32(0.35)$ & 0.36 & $-0.23(0.39)$ & 0.55 \\
\hline \multirow[t]{3}{*}{ Lactation } & 1 & $-1.62(0.27)$ & $<0.001$ & $0.34(0.16)$ & 0.04 \\
\hline & 2 & $-0.19(0.21)$ & 0.35 & $0.03(0.17)$ & 0.85 \\
\hline & 3 & Baseline & & Baseline & \\
\hline Vaccination & Vaccinated & $0.14(0.20)$ & 0.47 & $0.30(0.51)$ & 0.45 \\
\hline \multirow{4}{*}{ Vaccination $\times$ month in lactation } & 5 & NS & & Baseline & \\
\hline & 6 & NS & & $-0.34(0.64)$ & 0.60 \\
\hline & 7 & NS & & $-0.32(0.76)$ & 0.68 \\
\hline & 8 & NS & & $-1.57(0.68)$ & 0.02 \\
\hline \multirow[t]{2}{*}{ Herd } & $\mathrm{B}$ & $-2.84(0.28)$ & $<0.0001$ & $-0.89(0.14)$ & $<0.0001$ \\
\hline & $\mathrm{A}$ & Baseline & & Baseline & \\
\hline
\end{tabular}

${ }^{1}$ Only infections that occurred after the start of 50/50 randomization were used in this analysis. Herd was used as a random effect.

where the risk of new infection was significantly lower $(P<0.05)$ in 2 of the $8 \mathrm{mo}$ in lactation. Risk of new CNS IMI showed no pattern across month in milk or parity, with only parity 1 showing a lower new infection risk. A history of a previous CNS IMI turned out to be protective for the next new CNS IMI. Least squares means of the risk of new infections are shown for both Staph. aureus (Figure 3a) and CNS (Figure 3b).

Duration of infection was analyzed using KaplanMeier estimates of the survivor curve. The survivor curves are shown in Figure 4a for Staph. aureus and in Figure 4b for CNS. Using Cox regression, the estimation of hazard of curing an IMI by vaccination group resulted in a significantly increased hazard of ending the presence of infection in vaccinated versus control animals $(P<0.05)$. This was the case for both Staph. aureus and CNS (Table 3), but CNS IMI had a higher rate of cure, resulting in a shorter duration of infection for CNS compared with Staph. aureus (Table 3). Evidence for farm-specific patterns was also observed, with a higher risk of cure of Staph. aureus in farm B and a higher risk of cure of CNS in farm A.

Modeling Infection Dynamics. The monthly rate of new Staph. aureus infections was modeled in both herds using Poisson regression. First, it was evaluated whether evidence for contagious behavior of Staph. aureus existed by comparing a Poisson model with and without controlling for exposure to Staph. aureus (the offset term with and without). The difference between the model with and without controlling for Staph. aureus exposure was highly significant $(P<0.001)$, indicating that a very clear contagious component to Staph. aureus infection exists in both herds.

Modeling the effect of vaccination on the rate of new infections, correcting for the total exposure experience, indicated that vaccination status was statistically significant in an interaction with parity group. Vaccination was associated with a lower transmission parameter for new infections in lactation 1, a nonsignificant but numerically lower transmission parameter in parity 2 , and a significantly higher transmission parameter in lactations 3 and higher $(3+)$. In herd B, transmission of Staph. aureus was lower compared with herd A. These regression results are shown in Table 4.

Modeling the rate of cure of infection indicated that vaccination significantly increased the cure rate of Staph. aureus infections, this finding was consistent across lactation groups $(P<0.0001)$, but different between the 2 herds. Herd B had a significantly better rate of cure compared with herd A $(P<0.0001)$. These results are shown in Table 5.

The monthly rate of new CNS IMI was also modeled using Poisson regression. The difference between the model with and without including exposure to CNS was highly significant, indicating that a very clear contagious component to CNS infection exists in both 

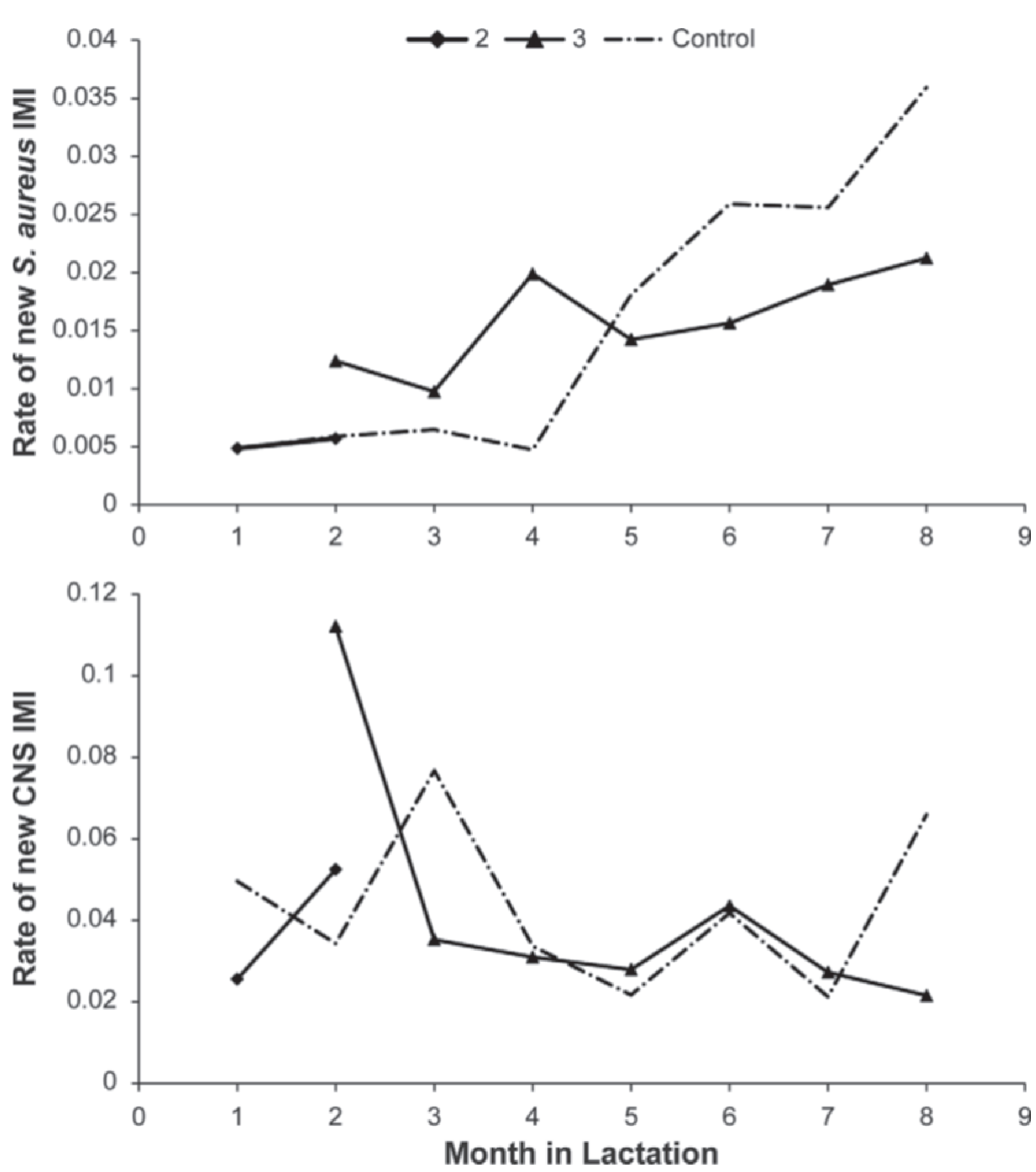

Figure 3. Incidence of new Staphylococcus aureus (top) and new CNS (bottom) IMI by months in lactation in vaccinated and control (dash-dotted line) cows. Cows that eventually were fully vaccinated were included in this analysis. As per vaccination protocol, vaccinated cows received 2 vaccinations at the start of lactation (2) and received the third and final dose (3) at approximately 53 DIM.

herds $(P<0.001)$. No difference in new infection rate of CNS was shown between vaccinated and control cows (Table 2).

A significantly lower transmission parameter was observed in vaccinated cows $(P<0.001)$ in both herds (Table 4). Comparing the 2 herds, farm $\mathrm{B}$ again showed a lower transmission parameter in CNS infections compared with farm A $(P<0.001)$. These results are shown in Table 4.

Modeling the rate of cure of CNS IMI indicated that vaccination significantly increased the cure rate of CNS infections $(P<0.001)$; this finding was consistent across the 2 herds. Comparing the 2 herds, farm A had a significantly better cure and, therefore, a shorter duration compared with farm $\mathrm{B}$. These results are shown in Table 5.

Combining the transmission parameter and cure rate parameter into the overall basic reproduction ratio, $R_{0}$, for Staph. aureus resulted in an $\mathrm{R}_{0}$ value of $0.89(95 \%$ $\mathrm{CI}=0.44-1.57)$ for vaccinated animals and a value of $1.72(95 \% \mathrm{CI}=1.06-3.17)$ for control cows. For CNS, the $R_{0}$ value for vaccinated animals was $0.91(95 \%$ CI $=0.78-1.14)$ and for control cows was $1.40(95 \% \mathrm{CI}=$ $1.16-1.70)$. 

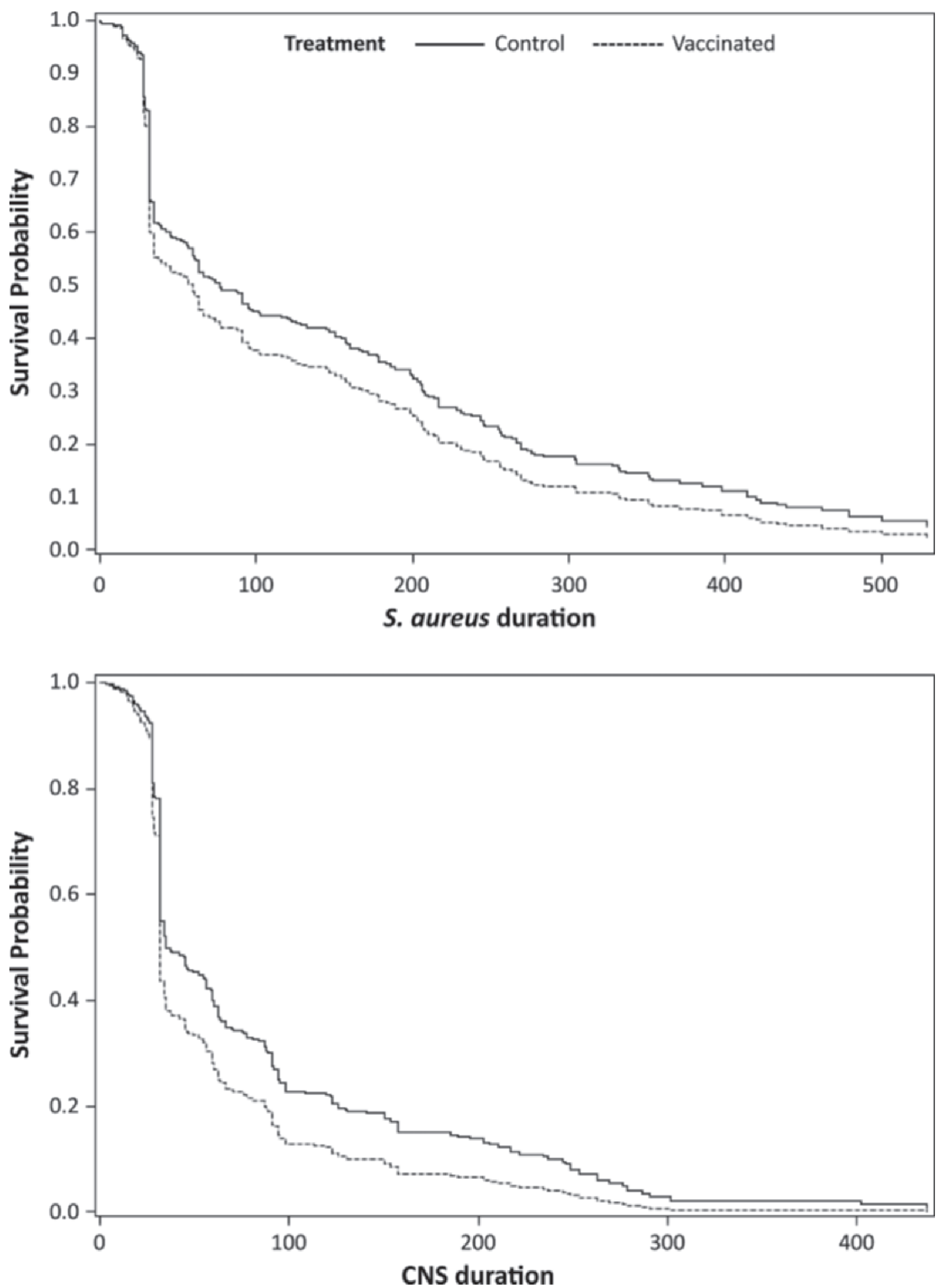

Figure 4. Survivor curves estimated from Cox time to event regression analysis. Shown here are time to cure of Staphylococcus aureus (top) and CNS (bottom) IMI.

\section{Vaccine Efficacy}

For the transmission parameters, $\alpha$ and $\beta$, the vaccine efficacy is shown in the Table 6 . The data indicated that vaccine efficacy for transmission is relatively low; in Staph. aureus it is either $25 \%$ in lactation 1 or nonsignificant but positive $(16 \%)$ and even negative $(-30 \%)$ in lactations 2 and $3+$, respectively. For CNS the transmission vaccine efficacy was $21 \%$, and this was consistent across lactations. Vaccine efficacy for cure is 
Table 3. Regression results of Cox time to event analyses ${ }^{1}$

\begin{tabular}{|c|c|c|c|c|c|c|}
\hline Parameter & & Estimate & $\mathrm{SE}$ & Chi-square & $P$-value & $\begin{array}{c}\text { Hazard } \\
\text { ratio }\end{array}$ \\
\hline \multicolumn{7}{|c|}{ Staphylococcus aureus } \\
\hline Vaccination & $\begin{array}{l}\text { Vaccinated } \\
\text { Control }\end{array}$ & $\begin{array}{c}0.24 \\
\text { Baseline }\end{array}$ & 0.12 & 3.88 & 0.05 & 1.27 \\
\hline Herd & $\begin{array}{l}\mathrm{B} \\
\mathrm{A}\end{array}$ & $\begin{array}{c}-0.61 \\
\text { Baseline }\end{array}$ & 0.12 & 26.74 & $<0.0001$ & 0.54 \\
\hline \multicolumn{7}{|l|}{ CNS } \\
\hline Herd & $\begin{array}{l}\mathrm{B} \\
\mathrm{A}\end{array}$ & $\begin{array}{c}0.25 \\
\text { Baseline }\end{array}$ & 0.08 & 9.13 & $<0.01$ & 1.28 \\
\hline
\end{tabular}

${ }^{1}$ Time to event here is time to cure for known newly infected quarters. Only infections occurring after the start of 50/50 randomization were used in this analysis. Results are shown for both Staph. aureus and CNS.

moderate, with a value of $41 \%$ for Staph. aureus and $16 \%$ for CNS. For Staph. aureus, a significant difference in vaccine efficacy for cure was present between the 2 farms; farm B showed a vaccine efficacy of cure of $52 \%$ and farm A was $30 \%$. When parameter estimates for transmission and cure were combined into a vaccine efficacy for the basic reproduction ratio, then an efficacy of $33 \%$ was present for reduction in basic reproduction ratio in CNS across herds and across lactations. For Staph. aureus, vaccine efficacy was $56 \%$ for lactation 1 animals, approximately $50 \%$ for lactation 2 animals, and dropped further to $24 \%$ in lactation $3+$ animals. When estimated across all animals in both herds, efficacy of vaccination with regard to the basic reproduction ratio for Staph. aureus was $45 \%$.

\section{DISCUSSION}

The key finding of the present study was a first estimate of the population efficacy of a staphylococcal vaccine affecting the transmission of infection. The ob- served reduction in the basic reproduction ratio, $\mathrm{R}_{0}$, of approximately $35 \%$ in CNS and $45 \%$ in Staph. aureus, is encouraging, but at the same time highlights that vaccination is only an additional tool in the control of staphylococcal infections on dairy farms. For both CNS and Staph. aureus, vaccination resulted in moving the basic reproduction ratio from above to below the threshold of one. This basic reproduction ratio, with a value below 1, points toward a fade-out of infection in vaccinated groups. However, elimination of infection from a farm or a group of cows would only be possible when no new infection from sources other than shedding herd mates occurs; in reality, infections from other sources are quite likely (Reksen et al., 2012; Barlow et al., 2013). In addition, the variability in this estimate would allow individual farms to continue to have endemic staphylococcal IMI despite vaccination. Such variation in a basic reproduction ratio of staphylococcal IMI has been observed before and was related to known infection risk factors (Lam et al., 1996, 1997; Reksen et al., 2012).

Table 4. Modeling the transmission parameter $\left(\beta^{*}\right)$ using the rate of new Staphylococcus aureus and CNS infections in a Poisson regression model ${ }^{1}$

\begin{tabular}{|c|c|c|c|c|c|}
\hline \multirow[b]{2}{*}{ Effect } & \multirow[b]{2}{*}{ Category } & \multicolumn{2}{|c|}{ Staph. aureus } & \multicolumn{2}{|l|}{ CNS } \\
\hline & & Estimate (SE) & $\operatorname{Pr}>|t|$ & Estimate (SE) & $\operatorname{Pr}>|t|$ \\
\hline$\beta^{*}$ & & $-1.22(0.10)$ & $<0.0001$ & $-0.25(0.08)$ & 0.00 \\
\hline Herd & B & $-0.21(0.10)$ & 0.03 & $-0.46(0.07)$ & $<0.0001$ \\
\hline Lactation group & $\begin{array}{l}1 \\
2 \\
3+\end{array}$ & $\begin{array}{l}0.05(0.19) \\
-0.18(0.14) \\
\text { Baseline }\end{array}$ & $\begin{array}{l}0.80 \\
0.20\end{array}$ & $\begin{array}{r}-0.24(0.08) \\
0.11(0.09)\end{array}$ & $\begin{array}{l}0.00 \\
0.23\end{array}$ \\
\hline Vaccine & $\begin{array}{l}\text { Vaccinated } \\
\text { Control }\end{array}$ & $\begin{array}{l}0.26(0.15) \\
\text { Baseline }\end{array}$ & 0.08 & $-0.22(0.07)$ & 0.00 \\
\hline Vaccine $\times$ lactation group & $\begin{array}{l}\text { Vaccinated } \times 1 \\
\text { Vaccinated } \times 2 \\
\text { Vaccinated } \times 3+\end{array}$ & $\begin{array}{l}-0.54(0.23) \\
-0.43(0.20) \\
\text { Baseline }\end{array}$ & $\begin{array}{l}0.02 \\
0.03\end{array}$ & $\begin{array}{l}\text { NS } \\
\text { NS } \\
\text { NS }\end{array}$ & \\
\hline Months since $50 / 50$ & & $0.02(0.01)$ & 0.01 & $0.01(0.01)$ & 0.25 \\
\hline
\end{tabular}

${ }^{1}$ The unit of analysis in this model is a calendar month. All months during the trial are included in the analysis; month since 50/50 vaccination was forced into the model as a covariate. 
Table 5. Modeling the cure parameter $\left(\alpha^{*}\right)$ using rate of cure

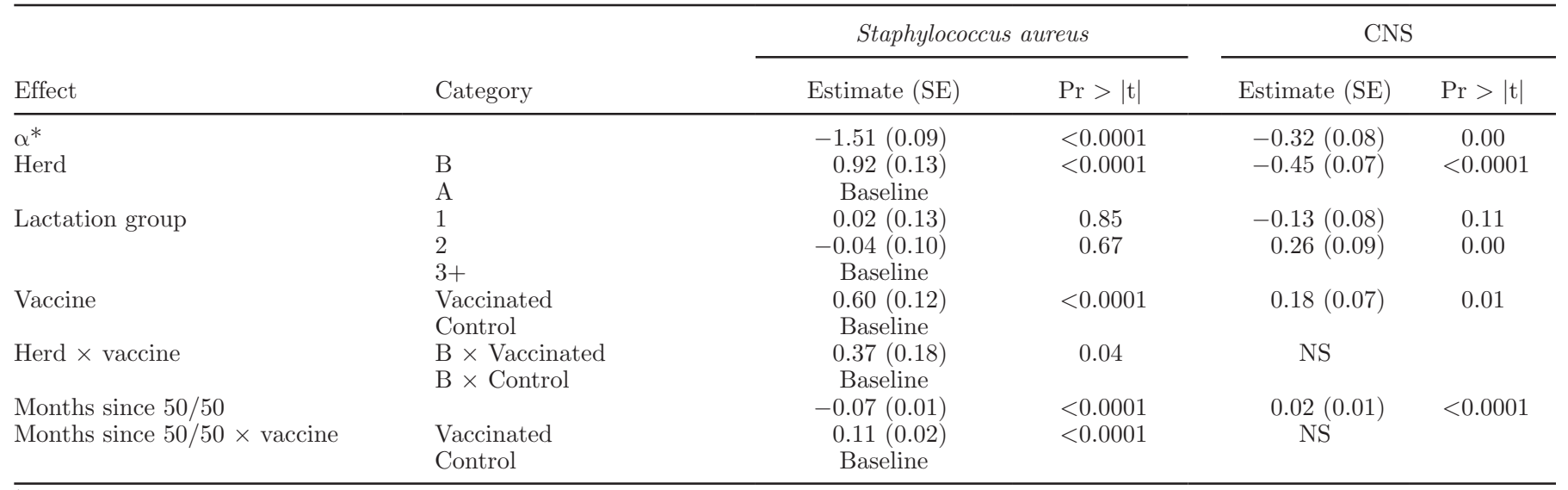

${ }^{1}$ The unit of analysis in this model is a calendar month. All months during the trial are included in the analysis; month since 50/50 vaccination was forced into the model as a covariate.

For the analysis of the data, we focused our efficacy estimates on cows that received a full vaccination protocol. Despite the ambition to vaccinate cows according to the label vaccination scheme, this was not always easy. Inaccurate estimation of duration of pregnancy, early loss of pregnancy, abortions, and cows calving early were the most important reasons for incomplete vaccination. Given that incomplete vaccination due to the reasons described here is a reality in many herds, it is possible that our estimates of vaccine efficacy are overestimated compared with the truly observed vaccine efficacy under field conditions.

A novel contribution of the current study was the chosen design of the study. By first fully vaccinating all cows before calving it was possible to reach a state of more or less equal groups of vaccinated and control cows. In herds with a year-round calving pattern it may be expected that $50 \%$ of cows calve at approximately 6 mo. After reaching $6 \mathrm{mo}$, vaccination was randomized at 50/50. At the time of starting the 50/50 randomization, the cohort of unvaccinated cows was later in lactation compared with the vaccinated cows, hence the proportion of vaccinated cows will initially increase to approximately $65 \%$ before falling back to $50 \%$. This increase will be lower when the time to $50 / 50$ is longer, as the difference in lactation stage in the fully vaccinated and control cohorts will be smaller. These effects can be seen in Figure 1. A deviation in ratio of vaccinates versus controls above 1 will result in a slight increase in power, as the expected number of events will be lower in the vaccinated arm of the study (Dohoo, 2004). In contrast, when 50/50 vaccination had been started immediately, it would have taken approximately $1.5 \mathrm{yr}$ before the 50/50 ratio would have been obtained in the full lactating herd.

The chosen study design, with comingling of vaccinated and control cows, allowed us to estimate population vaccine efficacy within herd using a within-herd randomization schedule. The difference between estimates of direct vaccine effects using simple regression analysis and population vaccine efficacy using a more elaborate mathematical modeling approach was large. Simple regression, resulting in an estimate of direct vaccine efficacy, showed no significant effects of vac-

Table 6. Estimation of vaccine efficacy with regard to infection transmission, infection cure, and basic reproduction ratio

\begin{tabular}{llcc}
\hline Parameter & Category & $\begin{array}{c}\text { Staphylococcus } \\
\text { aureus }\end{array}$ & CNS \\
\hline$\beta$, transmission & Lactation 1 & 0.25 & 0.20 \\
& Lactation 2 & 0.16 & 0.20 \\
$\alpha$, cure & Lactation 3 & -0.30 & 0.20 \\
& Herd B & 0.52 & \\
$\mathrm{R}_{0}$, basic reproduction ratio & Herd A & 0.30 & 0.16 \\
& All & 0.41 & 0.33 \\
& Lactation 1 & 0.56 & 0.33 \\
& Lactation 2 & 0.50 & 0.33 \\
& Lactation 3 & 0.24 & 0.33 \\
\hline
\end{tabular}


cination on new infections. Simple regression analysis without controlling for exposure environment will provide an estimate of direct vaccine efficacy, whereas our mathematical modeling resulted in an unbiased estimated of overall population vaccine efficacy (Halloran et al., 1997). The estimate of direct vaccine efficacy in comingled populations is expected to be biased toward no effect (Halloran et al., 1998, 2008). Therefore, as expected, the estimates of population vaccine efficacy were larger than the direct effect estimates. These population vaccine efficacy estimates were likely unbiased when proper control for total exposure experience was included. Related to the significant overall population efficacy of vaccination was the finding that the inclusion of total exposure experience in modeling of new infections was highly significant for both Staph. aureus and CNS. The very same finding was reported for Staph. aureus previously by Lam et al. (1996) and Barlow et al. (2013). Modeling infection transmission of CNS was reported by Reksen et al. (2012). In the current study, inclusion of total exposure experience was significant on 1 farm, but not important on a second farm. Although CNS exposure experience was important in both farms in the current study, large differences existed between farms with regard to CNS infection dynamics. This difference between farms may be explained by the dominant CNS species that is present in each particular farm. Until now, CNS species were not determined on the isolates collected in the current study. Future work on the collected isolates may provide more insight.

Previous studies on staphylococcal vaccination in dairy cows, as reviewed by Middleton et al. (2009) and Pereira et al. (2011), have shown relatively low vaccine efficacy or no vaccine efficacy at all. However, all the reviewed studies only reported direct vaccine efficacy and did not attempt to estimate overall population estimates. As staphylococcal IMI are mostly subclinical, and reducing infection transmission is the key outcome of interest, we would argue that carefully designed field studies with appropriate population dynamics-based vaccine efficacy estimates are essential to allow unbiased vaccine efficacy estimates. The design and analyses that we report here may therefore be of interest for future studies on staphylococcal vaccine efficacy in dairy herds (Daum and Spellberg, 2012).

In the analysis of new infection risk with Staph. aureus, a significant interaction of month in lactation with vaccination group was observed, with an increased risk with increasing months in lactation. Similarly, risk of infection increased with lactation number. Vaccine effects were observed throughout all analyses that were performed. In the analyses that we performed, we observed a significant decrease in prevalence of Staph. aureus in vaccinated cows compared with control cows, particularly later in lactation (Figure 2a). Rate of new infection and prevalence of CNS tended to be lower in vaccinated cows compared with control cows (Figures $2 \mathrm{~b}$ and $3 \mathrm{~b}$ ). Duration of infection was significantly shorter in vaccinated cows compared with control cows for both Staph. aureus and CNS (Figure 4a and b). The observed reduction in duration will benefit the affected cows but will also reduce the overall herd exposure experience of the other cows in the herd, resulting in an important contribution to indirect vaccine efficacy (Halloran et al., 1997, 1998).

Diagnosing IMI is not straightforward. Classical culture of milk samples has a limited sensitivity, therefore diagnostic errors will occur. In the current study we used monthly samples of all quarters of all cows, resulting in an overall increased sensitivity to detect IMI. However, the precise duration of the IMI may be underestimated. Similarly, we defined cure- or culturenegative based on 2 subsequent samples, with the exception of the edges of the study (start and end) where single samples were sufficient to determine the absence of infection. We chose the latter definition to increase sensitivity in the diagnosis of cure, but obviously at the cost of reduced specificity. These diagnostic errors occur with equal probability in vaccinates and controls and are therefore not likely to bias the results of the study. Other simulation studies have shown that imprecise diagnosis of IMI in comparative studies generally results in a bias toward the null hypothesis of no effect (Morant et al., 1988).

Modeling infection dynamics showed a significantly lower transmission parameter and rate of cure parameter for CNS across farms and groups of cows. For Staph. aureus, a lower transmission parameter was present in first lactation animals, and this effect of vaccination decreased with increasing lactation number. It has been shown before that IMI with Staph. aureus in older cows are less easily influenced by interventions (Barkema et al., 2006), most likely due to chronicity of the infection.

The farms showed a large difference in their ability to control infection during the study. For both farms, the prevalence of CNS remained more or less constant, but this was due to a slight increase in the control group and a decrease in prevalence in the vaccination group. Prevalence of Staph. aureus remained the same or slightly increased in farm A but dropped dramatically to a very low prevalence in farm B. Farm B was also actively culling Staph. aureus cows at a higher rate compared with non-Staph. aureus cows $(P>0.05)$. The farm managers had no knowledge of the culture data during the trial, so the increase culling of Staph. aureus cows was based on the actual performance of the animals and not because of an increased culling policy for known Staph. aureus cows. Culling policy in farm 
$\mathrm{B}$ was mostly based on fertility, lameness, and milk production.

The observed vaccine efficacy may vary depending on farm-specific characteristics, such as strain types (Smith et al., 1998; Barlow et al., 2013) and farm management practices (Lam et al., 1997), as we identified significant differences between farms. The known farm management practices associated with infection dynamics of Staph. aureus included treatment protocols, segregation and culling of known infected animals, milking procedures, and milking equipment (Barkema et al., 2006; Olde Riekerink et al., 2010). These differences in farm management practices will likely also be involved in determining the observed efficacy of staphylococcal vaccinations on dairy farms. For example, on farms with good management practices, $\mathrm{R}_{0}$ for Staph. aureus would be reduced from 1.5 to 0.83 , whereas vaccination on farms with poor management would reduce $\mathrm{R}_{0}$ from 5 to 2.75. In the latter example, Staph. aureus would show a reduced prevalence but remain endemic despite vaccination, whereas, in the first example, Staph. aureus would eventually be eliminated due to vaccination. In the present study we did not perform a cost-benefit analysis of the vaccine regimen used in this field study. Eventually, such a cost-benefit analysis will be essential to decide under what infection conditions vaccination would be economically beneficial to the farm.

\section{CONCLUSIONS}

Vaccine efficacy was moderate in our field trial in 2 commercial dairy herds. Vaccination was able to reduce the basic reproduction ratio of CNS and Staph. aureus in both herds. The data indicated that vaccination will result in reduction of the basic reproduction ratio of Staph. aureus by approximately $45 \%$ and the basic reproduction ratio for CNS by approximately $35 \%$. Efficacy was dependent upon the age group of the animals, particularly for Staph. aureus, where first lactation animals showed a significantly higher value compared with animals in third and higher lactation. The observed vaccine efficacy may vary depending on farm management practices, as we identified significant differences between farms. Vaccination is a valuable tool in reducing incidence and prevalence of staphylococcal infection in herds. Vaccine utilization will need to be combined with excellent milking procedures, culling of known infected cattle, and other management procedures to effectively reduce incidence and duration of infection.

\section{ACKNOWLEDGMENTS}

We acknowledge the collaboration and support of the farm owners and their staff in the execution of this trial. The research in this study was financially supported by Hipra.

\section{REFERENCES}

Andersen, S., I. R. Dohoo, R. Olde Riekerink, H. Stryhn, and Mastitis Research Workers' Conference. 2010. Diagnosing intramammary infections: Evaluating expert opinions on the definition of intramammary infection using conjoint analysis. J. Dairy Sci. 93:2966-2975.

Barkema, H. W., Y. H. Schukken, and R. N. Zadoks. 2006. Invited review: The role of cow, pathogen, and treatment regimen in the therapeutic success of bovine Staphylococcus aureus mastitis. J. Dairy Sci. 89:1877-1895.

Barlow, J. W., R. N. Zadoks, and Y. H. Schukken. 2013. Effect of lactation therapy on Staphylococcus aureus transmission dynamics in two commercial dairy herds. BMC Vet. Res. 9:28-40.

Cha, E., D. Bar, J. A. Hertl, L. W. Tauer, G. Bennett, R. N. González, Y. H. Schukken, F. L. Welcome, and Y. T. Gröhn. 2011. The cost and management of different types of clinical mastitis in dairy cows estimated by dynamic programming. J. Dairy Sci. 94:4476-4487.

Daum, R. S., and B. Spellberg. 2012. Progress toward a Staphylococcus aureus vaccine. Clin. Infect. Dis. 54:560-567.

Dohoo, I. R. 2004. The design of randomized controlled trials of veterinary vaccines. Anim. Health Res. Rev. 5:235-238.

Dohoo, I. R., J. Smith, S. Andersen, D. F. Kelton, S. Godden, and Mastitis Research Workers' Conference. 2011. Diagnosing intramammary infections: Evaluation of definitions based on a single milk sample. J. Dairy Sci. 94:250-261.

Haber, M., I. M. Longini Jr., and M. E. Halloran. 1991. Measures of the effects of vaccination in a randomly mixing population. Int. J. Epidemiol. 20:300-310.

Halasa, T., K. Huijps, O. Østerås, and H. Hogeveen. 2007. Economic effects of bovine mastitis and mastitis management: A review. Vet. Q. 29:18-31.

Halloran, M. E., R. M. Anderson, R. S. Azevedo-Neto, W. J. Bellini, O. Branch, M. A. Burke, R. Compans, K. Day, L. Gooding, S. Gupta, J. Katz, O. Kew, H. Keyserling, R. Krause, A. A. Lal, E. Massad, A. R. McLean, P. Rosa, P. Rota, P. Wiener, S. G. Wynn, and D. M. Zanetta. 1998. Population biology, evolution, and immunology of vaccination and vaccination programs. Am. J. Med. Sci. 315:76-86.

Halloran, M. E., N. M. Ferguson, S. Eubank, I. M. Longini Jr., D. A. Cummings, B. Lewis, S. Xu, C. Fraser, A. Vullikanti, T. C. Germann, D. Wagener, R. Beckman, K. Kadau, C. Barrett, C. A. Macken, D. S. Burke, and P. Cooley. 2008. Modeling targeted layered containment of an influenza pandemic in the United States. Proc. Natl. Acad. Sci. USA 105:4639-4644.

Halloran, M. E., M. Haber, I. M. Longini Jr., and C. J. Struchiner. 1991. Direct and indirect effects in vaccine efficacy and effectiveness. Am. J. Epidemiol. 133:323-331.

Halloran, M. E., C. J. Struchiner, and I. M. Longini Jr. 1997. Study designs for evaluating different efficacy and effectiveness aspects of vaccines. Am. J. Epidemiol. 146:789-803.

Harro, J. M., B. M. Peters, G. A. O'May, N. Archer, P. Kerns, R. Prabhakara, and M. E. Shirtliff. 2010. Vaccine development in Staphylococcus aureus: Taking the biofilm phenotype into consideration. FEMS Immunol. Med. Microbiol. 59:306-323.

Hertl, J. A., Y. H. Schukken, D. Bar, G. J. Bennett, R. N. González, B. J. Rauch, F. L. Welcome, L. W. Tauer, and Y. T. Gröhn. 2011. The effect of recurrent episodes of clinical mastitis caused by gram-positive and gram-negative bacteria and other organisms on mortality and culling in Holstein dairy cows. J. Dairy Sci. 94:4863-4877.

ISO (International Organization for Standardization). 2007. Milking machine installations-Mechanical tests. Method 6690-2007. ISO, Geneva, Switzerland.

Lam, T. J., M. C. DeJong, Y. H. Schukken, and A. Brand. 1996. Mathematical modeling to estimate efficacy of postmilking teat disinfection in split-udder trials of dairy cows. J. Dairy Sci. 79:62-70. 
Lam, T. J., J. H. van Vliet, Y. H. Schukken, F. J. Grommers, A. van Velden-Russcher, H. W. Barkema, and A. Brand. 1997. The effect of discontinuation of postmilking teat disinfection in low somatic cell count herds. II. Dynamics of intramammary infections. Vet. Q. 19:47-53.

Lu, Z., Y. T. Grohn, R. L. Smith, D. R. Wolfgang, J. A. Van Kessel, and Y. H. Schukken. 2009. Assessing the potential impact of Salmonella vaccines in an endemically infected dairy herd. J. Theor. Biol. 259:770-784.

Middleton, J. R., C. D. Luby, and D. S. Adams. 2009. Efficacy of vaccination against staphylococcal mastitis: A review and new data. Vet. Microbiol. 134:192-198.

Morant, S. V., F. H. Dodd, and R. P. Natzke. 1988. Consequences of diagnostic errors in mastitis therapy trials. J. Dairy Res. 55:315329 .

NMC (National Mastitis Council). 1999. Laboratory Handbook on Bovine Mastitis. National Mastitis Council, Madison, WI.

Olde Riekerink, R. G., H. W. Barkema, D. T. Scholl, D. E. Poole, and D. F. Kelton. 2010. Management practices associated with the bulk-milk prevalence of Staphylococcus aureus in Canadian dairy farms. Prev. Vet. Med. 97:20-28.

Pereira, U. P., D. G. Oliveira, L. R. Mesquita, G. M. Costa, and L. J. Pereira. 2011. Efficacy of Staphylococcus aureus vaccines for bovine mastitis: A systematic review. Vet. Microbiol. 148:117-124.

Pérez, M. M., A. Prenafeta, J. Valle, J. Penadés, C. Rota, C. Solano, J. Marco, M. J. Grilló, I. Lasa, J. M. Irache, T. Maira-Litran, J. Jiménez-Barbero, L. Costa, G. B. Pier, D. de Andrés, and B. Amorena. 2009. Protection from Staphylococcus aureus mastitis associated with poly-N-acetyl beta-1,6 glucosamine specific antibody production using biofilm-embedded bacteria. Vaccine 27:23792386.

Prenafeta, A., R. March, A. Foix, I. Casals, and L. Costa. 2010. Study of the humoral immunological response after vaccination with a Staphylococcus aureus biofilm-embedded bacterin in dairy cows: Possible role of the exopolysaccharide specific antibody production in the protection from Staphylococcus aureus induced mastitis. Vet. Immunol. Immunopathol. 134:208-217.

Reksen, O., Y. T. Gröhn, J. W. Barlow, and Y. H. Schukken. 2012 Transmission dynamics of intramammary infections with coagulase-negative staphylococci. J. Dairy Sci. 95:4899-4910.

Schukken, Y. H., J. Günther, J. Fitzpatrick, M. C. Fontaine, L. Goetze, O. Holst, J. Leigh, W. Petzl, H. J. Schuberth, A. Sipka, D G. Smith, R. Quesnell, J. Watts, R. Yancey, H. Zerbe, A. Gurjar, R. N. Zadoks, H. M. Seyfert, and Members of the Pfizer Mastitis Research Consortium. 2011. Host-response patterns of intramammary infections in dairy cows. Vet. Immunol. Immunopathol. 144:270-289.

Smith, T. H., L. K. Fox, and J. R. Middleton. 1998. Outbreak of mastitis caused by one strain of Staphylococcus aureus in a closed dairy herd. J. Am. Vet. Med. Assoc. 212:553-556.

Torvaldsen, S., and P. B. McIntyre. 2002. Observational methods in epidemiologic assessment of vaccine effectiveness. Commun. Dis. Intell. Q. Rep. 26:451-457.

Wilson, D. J., B. A. Mallard, J. L. Burton, Y. H. Schukken, and Y. T. Gröhn. 2007. Milk and serum J5-specific antibody responses, milk production change, and clinical effects following intramammary Escherichia coli challenge for J5 vaccinate and control cows. Clin. Vaccine Immunol. 14:693-699. 